Article

\title{
Cosine Measures of Linguistic Neutrosophic Numbers and Their Application in Multiple Attribute Group Decision-Making
}

\author{
Lilian Shi * and Jun Ye \\ Department of Electrical and Information Engineering, Shaoxing University, 508 Huancheng West Road, \\ Shaoxing 312000, China; yehjun@aliyun.com \\ * Correspondence: cssll@usx.edu.cn; Tel.: +86-575-8834-2802
}

Received: 21 August 2017; Accepted: 19 September 2017; Published: 22 September 2017

\begin{abstract}
The linguistic neutrosophic numbers (LNNs) can express the truth, indeterminacy, and falsity degrees independently by three linguistic variables. Hence, they are an effective tool for describing indeterminate linguistic information under linguistic decision-making environments. Similarity measures are usual tools in decision-making problems. However, existing cosine similarity measures have been applied in decision-making problems, but they cannot deal with linguistic information under linguistic decision-making environments. To deal with the issue, we propose two cosine similarity measures based on distance and the included angle cosine of two vectors between LNNs. Then, we establish a multiple attribute group decision-making (MAGDM) method based on the cosine similarity measures under an LNN environment. Finally, a practical example about the decision-making problems of investment alternatives is presented to demonstrate the effective applications of the proposed MAGDM method under an LNN environment.
\end{abstract}

Keywords: cosine similarity measure; linguistic neutrosophic number; multiple attribute group decision-making

\section{Introduction}

The fuzzy decision-making method is an important and complex research topic in decision-making theory. In recent decades, various fuzzy decision-making methods have been presented and applied in many decision-making fields. However, in real-world situations, some complex decision-making problems cannot be described by evaluation information with real numbers. In general, decision-makers make decisions under circumstances with vague, imprecise, and uncertain information. Therefore, they prefer to make a qualitative evaluation for attributes using linguistic terms because of the complexity of objective things and the ambiguity of human thinking. For this reason, Zadeh firstly proposed the concept of a linguistic variable and its application to approximate reasoning [1]. Based on the concept of a linguistic variable, Herrera et al. put forward a consensus model in group decision making and established three steps for solving a multi-criteria decision-making problem under linguistic information $[2,3]$. Next, many scholars also provided some 2-tuple linguistic representation models [4-7], two-dimension uncertain linguistic operations [8-10], and aggregation operators [11-15] to deal with decision-making problems with linguistic information.

Furthermore, linguistic variables were integrated with other fuzzy theories to handle decision-making problems. Wang and Li proposed the aggregation operators of intuitionistic linguistic fuzzy numbers (ILFNs) and gave a decision-making approach by combining intuitionistic fuzzy numbers (IFNs) with linguistic variables [16]. Then, some extensions of IFNs were widely studied, including some improved intuitionistic linguistic aggregation operators and their application in MAGDM problems [17-23], interval-valued intuitionistic linguistic sets and their application in multi-criteria decision-making 
(MCDM) problems [24], and so on. Rodriguez et al. also proposed hesitant fuzzy linguistic term sets (HFLTSs) by combining the hesitant fuzzy sets (HFSs) with linguistic variables [25]. Some new HFLTS approaches have been presented to solve decision-making problems in recent years [26-34]. Moreover, Zhou et al. defined intuitionistic hesitant linguistic sets (IHLSs) and several operators on the basis of hesitant fuzzy linguistic sets (HFLSs) and intuitionistic linguistic sets (ILSs) [35]. Faizi et al. proposed an outranking method for MCDM problems using IHLSs [36].

In these extensions of fuzzy linguistic methods mentioned above, including ILFNs, HFLTSs, and IHLSs, there are only two linguistic variables for describing the linguistic information of both the truth/membership and falsity/non-membership degrees. So, the existing linguistic variables cannot express indeterminate or inconsistent linguistic information. However, the real decision-making problems often contain indeterminate or inconsistent information. To express this indeterminate or inconsistent information, Smarandache proposed the concept of neutrosophic sets (NSs) [37] and neutrosophic numbers (NNs) [38,39]. In neutrosophic theory, NSs and NNs are two different branches. Recently, NSs have been studied by some scholars and applied for solving MCDM problems [40-42]. NNs can effectively describe incomplete or indeterminate information because they consist of a determinate part and indeterminate part. Ye proposed a bidirectional projection method for MAGDM problems with NNs [43]. Ye presented a MAGDM method under an NN environment [44]. Linguistic variables can easily express a qualitative evaluation for an attribute in complex decision-making problems, but they cannot describe indeterminate or inconsistent evaluation information. To overcome the insufficiency of the existing linguistic variables, many researches extended linguistic variables to NSs and NNs. Li et al. introduced the concept of linguistic neutrosophic sets (LNSs) and their application in multicriteria decision-making problems [45]. Luo et al. proposed a decision-making approach based on an extended linguistic preference structure [46]. Wang et al. presented a decision-making method under single-valued neutrosophic linguistic environments [47]. Fang and Ye [48] put forward the concept of linguistic neutrosophic numbers (LNNs) by combining neutrosophic numbers (NNs) with linguistic variables, which is characterized independently by the truth, indeterminacy, and falsity linguistic variables. The basic operational laws of LNNs were developed. Further, an LNN-weighted arithmetic averaging (LNNWAA) operator and an LNN-weighted geometric averaging (LNNWGA) operator were proposed for MAGDM problems with LNNs [48]. The similarity measure between fuzzy sets is an important mathematical tool for determining the degree of similarity between two objects, which is effectively applied in decision-making problems. Biswas et al. proposed a cosine similarity measure of trapezoidal fuzzy neutrosophic numbers [49]. Mahmood et al. presented three similarity measures between simplified neutrosophic hesitant fuzzy sets [50]. The above cosine similarity measures have been applied in decision-making problems, but they cannot deal with linguistic information under linguistic decision-making environments. Hence, this paper extends cosine similarity measures to LNNs, which can solve linguistic information decision-making problems. In this paper, we propose two cosine similarity measures based on distance and the included angle cosine between LNNs in vector space, and establish a MAGDM method based on the cosine similarity measures under an LNN environment. The main advantage of the LNN method is that it is able to effectively handle indeterminate linguistic information under linguistic decision-making environments.

The rest of the article is organized as follows. Section 2 briefly introduces some basic concepts of LNNs. Section 3 proposes the cosine similarity measures based on distance and the included angle cosine of two vectors. In Section 4, we establish a MAGDM method based on the cosine similarity measures of LNNs. Section 5 gives a practical example and comparison analysis using LNNs. Conclusions of this work are summarized in Section 6.

\section{Some Basic Concepts of LNNs}

In this section, we briefly introduce basic concepts of linguistic variables, linguistic term sets, and linguistic neutrosophic numbers (LNNs), which will be needed in the following analysis. 
Zadeh firstly proposed the concept of linguistic variables [1], which represent qualitative data using words or sentences in natural language [2]. Let $S=\left\{s_{0}, s_{1}, \ldots, s_{\tau}\right\}$ is a linguistic term set with odd cardinality $\tau+1$, where $s_{i}$ represents the value of a linguistic variable. For example, taking $\tau=8$, one can specify a linguistic term set $S=\left\{s_{0}=\right.$ extremely low, $s_{1}=$ very low, $s_{2}=$ low, $s_{3}=$ slightly low, $s_{4}$ $=$ medium, $s_{5}=$ slightly high, $s_{6}=$ high, $s_{7}=$ very high, $s_{8}=$ extremely high $\}$. Then, the linguistic term set must satisfy the following characteristics [2,3]:

(1) Ordering: $s_{i} \geq s_{j}$ if $i \geq j$.

(2) Negation operator: $\operatorname{Neg}\left(s_{i}\right)=s_{\tau+1-i}$.

(3) Maximum operator: $\operatorname{Max}\left(s_{i}, s_{j}\right)=s_{i}$ if $i \geq j$.

(4) Minimum operator: $\operatorname{Min}\left(s_{i}, s_{j}\right)=s_{j}$ if $i \geq j$.

By combining neutrosophic numbers (NNs) [39,40] with linguistic variables, Fang and Ye [48] introduced the concept of linguistic neutrosophic numbers (LNNs) and give the following definition:

Definition 1 [48]. Assume that $S=\left\{s_{0}, s_{1}, \ldots, s_{\tau}\right\}$ is a linguistic term set with odd cardinality $\tau+1$. If $e=\left\langle s_{\alpha}\right.$, $s_{\beta}, s_{\gamma}>$ is defined for $s_{\alpha}, s_{\beta}, s_{\gamma} \in S$ and $\alpha, \beta, \gamma \in[0, \tau]$, where $s_{\alpha}, s_{\beta}$, and $s_{\gamma}$ represent, respectively, the truth degree, indeterminacy degree, and falsity degree by linguistic terms, then e is called a linguistic neutrosophic number (LNN).

\section{Cosine Measures of LNNs}

In this section, two cosine measures between LNNs are proposed.

Definition 2. Assume that $S=\left\{s_{0}, s_{1}, \ldots, s_{\tau}\right\}$ is a linguistic term set with odd cardinality $\tau+1$. If $E$ $=\left\{e_{1}, e_{2}, \ldots, e_{n}\right\}$ and $G=\left\{g_{1}, g_{2}, \ldots, g_{n}\right\}$ are two sets of LNNs, where $e_{k}=<s_{\alpha e_{k}}, s_{\beta e_{k_{k}}}, s_{\gamma e_{k}}>$ and $\mathrm{g}_{k}=<s_{\alpha g_{k}}, s_{\beta g_{k}}, s_{\gamma g_{k}}>$ are LNNs with, $s_{\beta e_{k}}, s_{\gamma e_{k}}, s_{\alpha g_{k}}, s_{\beta g_{k}}, s_{\gamma g_{k}} \in S$ and $f\left(s_{j}\right)=j$ is a linguistic scale function for $\alpha e_{k}, \beta e_{k}, \gamma e_{k}, \alpha g_{k}, \beta g_{k}, \gamma g_{k} \in[0, \tau]$ and $k=1,2, \ldots, n$. Then, two cosine measures of $E$ and $G$ are proposed based on distance and the included angle cosine of two vectors, respectively, as follows:

Cosine similarity measure based on distance

$$
\begin{aligned}
C^{1}{ }_{L N N S}(E, G) & =\frac{1}{n} \sum_{k=1}^{n} \cos \left(\frac{\left|f\left(s_{\alpha e_{k}}\right)-f\left(s_{\alpha g_{k}}\right)\right|+\left|f\left(s_{\beta e_{k}}\right)-f\left(s_{\beta g_{k}}\right)\right|+\left|f\left(s_{\gamma e_{k}}\right)-f\left(s_{\gamma g_{k}}\right)\right|}{6 \tau} \pi\right) \\
& =\frac{1}{n} \sum_{k=1}^{n} \cos \left(\frac{\left|\alpha e_{k}-\alpha g_{k}\right|+\left|\beta e_{k}-\beta g_{k}\right|+\left|\gamma e_{k}-\gamma g_{k}\right|}{6 \tau} \pi\right)
\end{aligned}
$$

Cosine similarity measure based on the included angle cosine of two vectors

$$
\begin{aligned}
& C^{2}{ }_{L N N S}(E, G)=\frac{1}{n} \sum_{k=1}^{n} \frac{f\left(s_{\alpha e_{k}}\right) \cdot f\left(s_{\alpha g_{k}}\right)+f\left(s_{\beta e_{k}}\right) \cdot f\left(s_{b_{g_{k}}}\right)+f\left(\mathrm{~s}_{\gamma e_{k}}\right) \cdot f\left(s_{\gamma g_{k}}\right)}{\sqrt{\left(f\left(s_{\alpha e_{k}}\right)\right)^{2}+\left(f\left(s_{\beta e_{k}}\right)\right)^{2}+\left(f\left(s_{\gamma e_{k}}\right)\right)^{2} \times \sqrt{\left(f\left(s_{\gamma g_{k}}\right)\right)^{2}+\left(f\left(s_{\beta g_{k}}\right)\right)^{2}+\left(f\left(s_{\gamma g_{k}}\right)\right)^{2}}}} \\
& =\frac{1}{n} \sum_{k=1}^{n} \frac{\alpha e_{k} \cdot \alpha g_{k}+\beta e_{k} \cdot \beta g_{k}+\gamma e_{k} \cdot \gamma g_{k}}{\sqrt{\left(\alpha e_{k}\right)^{2}+\left(\beta e_{k}\right)^{2}+\left(\gamma e_{k}\right)^{2}} \times \sqrt{\left(\alpha g_{k}\right)^{2}+\left(\beta g_{k}\right)^{2}+\left(\gamma g_{k}\right)^{2}}}
\end{aligned}
$$

According to the above definition, the two cosine similarity measures $C_{L N N s}^{i}(E, G)(i=1,2)$ for LNNs satisfy the following properties (p1)-(p3):

(p1) $0 \leq C_{L N N S}^{i}(E, G) \leq 1$;

(p2) $C_{L N N S}^{i}(E, G)=C_{L N N S}^{i}(G, E)$;

(p3) If $E=G$, then $C_{L N N s}^{i}(G, E)=1$.

Proof. Firstly, we prove the properties (p1)-(p3) of $C^{1}{ }_{L N N s}(E, G)$. 
(p1) Let $\theta=\left(\left|\alpha e_{k}-\alpha g_{k}\right|+\left|\beta e_{k}-\beta g_{k}\right|+\left|\gamma e_{k}-\gamma g_{k}\right|\right) \pi / 6 \tau$, there exist $0 \leq\left(\left|\alpha e_{k}-\alpha g_{k}\right|\right) / \tau \leq 1$, $0 \leq\left(\left|\beta e_{k}-\beta g_{k}\right|\right) / \tau \leq 1$, and $0 \leq\left(\left|\gamma e_{k}-\gamma g_{k}\right|\right) / \tau \leq 1$. Then, there is $0 \leq\left(\left|\alpha e_{k}-\alpha g_{k}\right|+\left|\beta e_{k}-\beta g_{k}\right|+\left|\gamma e_{k}-\gamma g_{k}\right|\right) / 3 \tau \leq 1$.

For $0 \leq \theta \leq \pi / 2,0 \leq \cos \theta \leq 1$. Hence, $0 \leq C^{1}{ }_{L N N s}(E, G) \leq 1$ holds.

(p2) It is straightforward.

(p3) If $E=G$, there are $\left\langle s_{\alpha e_{k}}, s_{\beta e_{k^{\prime}}}, s_{\gamma e_{k}}>=<s_{\alpha g_{k}}, s_{\beta g_{k}}, s_{\gamma g_{k}}>\right.$. Here, $E$ and $G$ can be considered as sets, there exist $E \supseteq G$ and $E \subseteq G$, then $s_{\alpha e_{k}}=s_{\alpha g_{k}}, s_{\beta e_{k}}=s_{\beta g_{k}}$ and $s_{\gamma e_{k}}=s_{\gamma g_{k}}$, for $k=1,2, \ldots, n$. According to the operational laws of LNNs, we have $f\left(s_{\alpha e_{k}}\right)=f\left(s_{\alpha g_{k}}\right)$, $f\left(s_{\beta e_{k}}\right)=f\left(s_{\beta g_{k}}\right)$, and $f\left(s_{\gamma e_{k}}\right)=f\left(s_{\gamma g_{k}}\right)$, hence $\alpha e_{k}=\alpha g_{k}, \beta e_{k}=\beta g_{k}$, and $\gamma e_{k}=\gamma g_{k}$, then $\cos \left\{\left(\left|\alpha e_{k}-\alpha g_{k}\right|+\left|\beta e_{k}-\beta \mathrm{g}_{k}\right|+\left|\gamma e_{k}-\gamma g_{k}\right|\right) \pi / 6 \tau\right\}=\cos 0=1$. Thus, $C^{1}{ }_{L N N s}(G, E)=1$ holds.

Secondly, we prove the properties (p1)-(p3) of $C^{2}{ }_{L N N S}(E, G)$.

(p1) It is obvious that $C^{2}{ }_{L N N s}(E, G) \geq 0$. Then, we only prove $C^{2}{ }_{L N N s}(E, G) \leq 1$.

According to Cauchy-Schwarz inequality, we can obtain the following inequality:

$$
\alpha e_{k} \cdot \alpha g_{k}+\beta e_{k} \cdot \beta g_{k}+\gamma e_{k} \cdot \gamma g_{k} \leq \sqrt{\left(\alpha e_{k}\right)^{2}+\left(\beta e_{k}\right)^{2}+\left(\lambda e_{k}\right)^{2}} \times \sqrt{\left(\alpha g_{k}\right)^{2}+\left(\beta g_{k}\right)^{2}+\left(\gamma g_{k}\right)^{2}} .
$$

Obviously, $\frac{1}{n} \sum_{k=1}^{n} \frac{\alpha e_{k} \cdot \alpha g_{k}+\beta e_{k} \cdot \beta g_{k}+\gamma e_{k} \cdot \gamma g_{k}}{\sqrt{\left(\alpha e_{k}\right)^{2}+\left(\beta e_{k}\right)^{2}+\left(\gamma e_{k}\right)^{2}} \times \sqrt{\left(\alpha g_{k}\right)^{2}+\left(\beta g_{k}\right)^{2}+\left(\gamma g_{k}\right)^{2}}} \leq 1$.

Hence, $0 \leq C^{2}{ }_{L N N S}(G, E) \leq 1$ holds.

(p2) It is obvious that the property is true.

(p3) If $E=G,<s_{\alpha e_{k}}, s_{\beta e_{k}}, s_{\gamma e_{k}}>=<s_{\alpha g_{k}}, s_{\beta g_{k}}, s_{\gamma g_{k}}>$, for $k=1,2, \ldots, n$. Here, $E$ and $G$ can be considered as two vectors, so $\|E\|=\|G\|,\|E\|=\sqrt{\left(f\left(s_{\alpha e_{k}}\right)\right)^{2}+\left(f\left(s_{\beta e_{k}}\right)\right)^{2}+\left(f\left(s_{\gamma e_{k}}\right)\right)^{2}}$, and $\|G\|=\sqrt{\left(f\left(s_{\alpha g_{k}}\right)\right)^{2}+\left(f\left(s_{\beta g_{k}}\right)\right)^{2}+\left(f\left(s_{\gamma g_{k}}\right)\right)^{2}}$, and there exists

$$
\begin{aligned}
& \frac{E \cdot G}{\|E\| \cdot\|G\|}=\frac{E \cdot E}{\|E\| \cdot\|G\|} \\
& =\frac{f\left(s_{\alpha e_{k}}\right) f\left(s_{\alpha g_{k}}\right)+f\left(s_{\beta e_{k}}\right) f\left(s_{\beta g_{k}}\right)+f\left(s_{\gamma e_{k}}\right) f\left(s_{\gamma g_{k}}\right)}{\sqrt{\left(f\left(s_{\alpha e_{k}}\right)\right)^{2}+\left(f\left(s_{\beta e_{k}}\right)\right)^{2}+\left(f\left(s_{\gamma e_{k}}\right)\right)^{2}} \cdot \sqrt{\left(f\left(s_{\alpha g_{k}}\right)\right)^{2}+\left(f\left(s_{\beta g_{k}}\right)\right)^{2}+\left(f\left(s_{\gamma g_{k}}\right)\right)^{2}}} \\
& =\frac{f\left(s_{\alpha e_{k}}\right) f\left(s_{\alpha g_{k}}\right)+f\left(s_{\beta e_{k}}\right) f\left(s_{\beta g_{k}}\right)+f\left(s_{\gamma e_{k}}\right) f\left(s_{\gamma g_{k}}\right)}{\left(f\left(s_{\alpha e_{k}}\right)\right)^{2}+\left(f\left(s_{\beta e_{k}}\right)\right)^{2}+\left(f\left(s_{\gamma e_{k}}\right)\right)^{2}} \\
& =\frac{\left(f\left(s_{\alpha e_{k}}\right)\right)^{2}+\left(f\left(s_{\beta e_{k}}\right)\right)^{2}+\left(f\left(s_{\gamma e_{k}}\right)\right)^{2}}{\left(f\left(s_{\alpha e_{k}}\right)\right)^{2}+\left(f\left(s_{\beta e_{k}}\right)\right)^{2}+\left(f\left(s_{\gamma e_{k}}\right)\right)^{2}}=1
\end{aligned}
$$

According to the operational laws of LNNs, we have $f\left(s_{\alpha e_{k}}\right)=\alpha e_{k}, f\left(s_{\beta e_{k}}\right)=\beta e_{k}, f\left(s_{\gamma e_{k}}\right)=\gamma e_{k}$, $f\left(s_{\alpha g_{k}}\right)=\alpha g_{k}, f\left(s_{\beta g_{k}}\right)=\beta g_{k}$, and $f\left(s_{\gamma e} g\right)=\gamma g_{k}$, then

$$
\frac{\alpha e_{k} \cdot \alpha g_{k}+\beta e_{k} \cdot \beta g_{k}+\gamma e_{k} \cdot \gamma g_{k}}{\sqrt{\left(\alpha e_{k}\right)^{2}+\left(\beta e_{k}\right)^{2}+\left(\gamma e_{k}\right)^{2}} \times \sqrt{\left(\alpha g_{k}\right)^{2}+\left(\beta g_{k}\right)^{2}+\left(\gamma g_{k}\right)^{2}}}=1
$$

Hence, $C^{2}{ }_{L N N S}(G, E)=1$ holds.

Thus, we have finished the proof.

If we consider the weights of the elements $e_{k}$ and $g_{k}(k=1,2, \ldots, n)$, the two weighted cosine similarity measures between $E$ and $G$ are proposed, respectively, as follows:

$$
\begin{gathered}
C^{\omega 1}{ }_{L N N S}(E, G)=\sum_{k=1}^{n} \omega_{k} \cos \left(\frac{\left|\alpha e_{k}-\alpha g_{k}\right|+\left|\beta e_{k}-\beta g_{k}\right|+\left|\gamma e_{k}-\gamma g_{k}\right|}{6 \tau} \pi\right) \\
C^{\omega 2}{ }_{L N N S}(E, G)=\sum_{k=1}^{n} \omega_{k} \frac{\alpha e_{k} \cdot \alpha g_{k}+\beta e_{k} \cdot \beta g_{k}+\gamma e_{k} \cdot \gamma g_{k}}{\sqrt{\left(\alpha e_{k}\right)^{2}+\left(\beta e_{k}\right)^{2}+\left(\gamma e_{k}\right)^{2}} \times \sqrt{\left(\alpha g_{k}\right)^{2}+\left(\beta g_{k}\right)^{2}+\left(\gamma g_{k}\right)^{2}}}
\end{gathered}
$$


where $\omega_{k} \in[0,1]$, and $\sum_{k=1}^{n} \omega_{k}=1$ for $k=1,2, \ldots, n$.

It is obvious that the two weighted cosine similarity measures $C^{\omega i}{ }_{L N N s}(E, G)(i=1,2)$ also satisfy the following properties (p1)-(p3):

(p1) $0 \leq C^{\omega i}{ }_{L N N s}(E, G) \leq 1$;

(p2) $C^{\omega i}{ }_{L N N s}(E, G)=C^{\omega i}{ }_{L N N S}(G, E)$;

(p3) If $E=G$, then $C^{\omega i}{ }_{L N N s}=1$.

Especially when $\omega_{k}=1 / n$ for $k=1,2, \ldots, n$, Equations (3) and (4) are reduced to Equations (1) and (2), respectively.

We can easily prove the properties (p1)-(p3) for $C^{\omega i}{ }_{L N N s}(E, G)(i=1,2)$ by a similar proof process.

\section{MAGDM Method Based on the Cosine Measures of LNNs}

In this section, we apply the cosine similarity measures of LNNs to solve MAGDM problems with LNN information.

For an MAGDM problem, let $H=\left\{h_{1}, h_{2}, \ldots, h_{m}\right\}$ be a set of $m$ alternatives and $A=\left\{A_{1}, A_{2}, \ldots, A_{n}\right\}$ be a set of $n$ attributes. The weight vector of the attributes $A_{j}(j=1,2, \ldots, n)$ is $\omega_{A}=\left(\omega_{A 1}, \omega_{A 2}, \ldots, \omega_{A n}\right)^{\mathrm{T}}$, satisfying $\omega_{A j} \in[0,1]$, and $\sum_{j=1}^{n} \omega_{A j}=1$ for $j=1,2, \ldots, n$. Assume that $E X=\left\{E X_{1}, E X_{2}, \ldots, E X_{y}\right\}$ is a group of experts and their corresponding weight vector is $\omega_{E}=\left(\omega_{E 1}, \omega_{E 2}, \ldots, \omega_{E y}\right)^{\mathrm{T}}$, satisfying $\omega_{E k} \in[0,1]$, and $\sum_{k=1}^{y} \omega_{E k}=1$ for $k=1,2, \ldots, y$. The linguistic term is set $S=\left\{s_{0}=\right.$ extremely low, $s_{1}=$ very low, $s_{3}=$ low, $s_{4}=$ medium, $s_{5}=$ slightly high, $s_{6}=$ high, $s_{7}=$ very high, $s_{8}=$ extremely high $\}$. Each expert can assign the truth degree, falsity degree, and indeterminacy degree to each attribute $A_{j}(j=1,2, \ldots, n)$ on the alternatives $h_{i}(i=1,2, \ldots, m)$ according to the linguistic terms, respectively. Therefore, we can established an LNN decision matrix $D^{k}=\left(d_{i, j}^{k}\right)_{m \times n}=\left[D_{1}^{k}, D_{2^{k}}^{k}, \cdots, D_{m}^{k}\right]^{\mathrm{T}}$, where $d_{i, j}^{k}=<s_{\alpha_{i, j}^{k},} s_{\beta_{i, j}^{k},} s_{\gamma_{i, j}^{k}}>(i=1,2, \ldots, m ; j=1,2$, $\ldots, n ; k=1,2, \ldots, y)$ is an LNN for $s_{\alpha_{i, j}^{k}}, s_{\beta_{i, j}^{k}}, s_{\gamma_{i, j}^{k}} \in[0,1]$, and $D_{i}^{k}=\left\{d_{i, 1}^{k}, d_{i, 2}^{k}, \ldots, d_{i, n}^{k}\right\}=$ $\left\{<s_{\alpha_{i, 1}^{k}}, s_{\beta_{i, 1}^{k}}, s_{\gamma_{i, 1}^{k}}>,<s_{\alpha_{i, 2}^{k}}, s_{\beta_{i, 2}^{k}}, s_{\gamma_{i, 2}^{k}}>, \ldots,<s_{\alpha_{i, n}^{k}}, s_{\beta_{i, n}^{k}}, s_{\gamma_{i, n}^{k}}>\right\}$.

Then, we apply the cosine similarity measures of LNNs to solve MAGDM problems using the following steps:

Step 1: Establish an ideal alternative (ideal solution) LNN matrix as follows: $H^{*}=\left(h_{i, j}^{*}\right)_{m \times n}=$ $\left[H_{1}^{*}, H_{2}^{*}, \cdots, H_{m}^{*}\right]^{\mathrm{T}}$ with $h_{i, j}^{*}=<\max \left(s_{\alpha_{i, j}}\right), \min \left(s_{\beta_{i, j}}\right), \min \left(s_{\gamma_{i, j}}\right)>(i=1,2, \ldots, m ; j=1,2, \ldots, n)$.

Step 2: Calculate the weighted cosine measure values between $D_{i}^{k}$ and the ideal alternative $H_{i}^{*}$ by Equation (3) or Equation (4) and obtain the value of $C^{\omega 1}{ }_{L N N s}\left(D_{i}^{k}, H_{i}^{*}\right)$ or $C^{\omega 2}{ }_{L N N s}\left(D_{i}^{k}, H_{i}^{*}\right)(i=1,2, \ldots, m)$.

Step 3: Calculate the overall weighted cosine measure values considering the corresponding weight of each expert to evaluate the alternatives $H_{i}(i=1,2, \ldots, m)$, as follows:

$$
\begin{aligned}
& C^{\omega 1}{ }_{L N N s}\left(D^{k}, H_{i}\right)=\sum_{k=1}^{y} \omega_{E k} \cdot C^{\omega 1}{ }_{L N N s}\left(D_{i}^{k}, H_{i}^{*}\right) \\
& C^{\omega 2}{ }_{L N N s}\left(D^{k}, H_{i}\right)=\sum_{k=1}^{y} \omega_{E k} \cdot C^{\omega 2}{ }_{L N N s}\left(D_{i}^{k}, H_{i}^{*}\right)
\end{aligned}
$$

where $\omega_{E k} \in[0,1]$ and $\sum_{k=1}^{y} \omega_{E k}=1$ for $k=1,2, \ldots, y$.

Step 4: Rank the alternatives according to the values of $C^{\omega 1}{ }_{L N N s}\left(D^{k}, H_{i}\right)$ or $C^{\omega 2}{ }_{L N N s}\left(D^{k}, H_{i}\right)$ and select the best one(s). The bigger the cosine measure value, the better the alternative.

Step 5: End. 


\section{Practical Example and Comparison Analysis}

In this section, we provide a practical example of the selection problem of investment alternatives adapted from [48] to demonstrate the applications of the developed MAGDM approach with neutrosophic linguistic information.

\subsection{Practical Example}

There is an investment company, which needs to invest a sum of money in the best selection. There is a panel with four possible investment alternatives $H=\left\{H_{1}, H_{2}, H_{3}, H_{4}\right\}$, where: (a) $H_{1}$ is a car company; (b) $H_{2}$ is a food company; (c) $H_{3}$ is a computer company; (d) $H_{4}$ is an arms company. The investment company needs to make a decision based on three attributes: (a) $A_{1}$ is the risk factor; (b) $A_{2}$ is the growth factor; (c) $A_{3}$ is the environmental factor. The weight vector of the three attributes is $\omega_{A}=(0.35,0.25,0.4)^{\mathrm{T}}$. Three decision-makers denoted as $E X=\left\{E X_{1}, E X_{2}, E X_{3}\right\}$ are invited to evaluate the alternatives on the three attributes by LNNs from the linguistic term set $S=\left\{s_{0}=\right.$ extremely low, $s_{1}=$ very low, $s_{3}=$ low, $s_{4}=$ medium, $s_{5}=$ slightly high, $s_{6}=$ high, $s_{7}=$ very high, $s_{8}=$ extremely high $\}$, and then their corresponding weight vector is given as $\omega_{E}=(0.37,0.33,0.3)^{\mathrm{T}}$. Thus, we can establish the LNN decision matrix $D^{k}$ according to the linguistic evaluation information given by each decision-maker $E X_{k}(k=1,2,3)$ as follows:

$$
\begin{gathered}
D^{1}=\left[\begin{array}{l}
D_{1}^{1} \\
D_{2}^{1} \\
D_{3}^{1} \\
D_{4}^{1}
\end{array}\right]=\left[\begin{array}{l}
<s_{6}, s_{1}, s_{2}><s_{7}, s_{2}, s_{1}><s_{6}, s_{2}, s_{2}> \\
<s_{7}, s_{1}, s_{1}><s_{7}, s_{3}, s_{2}><s_{7}, s_{2}, s_{1}> \\
<s_{6}, s_{2}, s_{2}><s_{7}, s_{1}, s_{1}><s_{6}, s_{2}, s_{2}> \\
<s_{7}, s_{1}, s_{2}><s_{7}, s_{2}, s_{3}><s_{7}, s_{2}, s_{1}>
\end{array}\right], \\
D^{2}=\left[\begin{array}{c}
D_{1}^{2} \\
D_{2}^{2} \\
D_{3}^{2} \\
D_{4}^{2}
\end{array}\right]=\left[\begin{array}{l}
<s_{6}, s_{1}, s_{2}><s_{6}, s_{1}, s_{1}><s_{4}, s_{2}, s_{3}> \\
<s_{7}, s_{2}, s_{3}><s_{6}, s_{1}, s_{1}><s_{4}, s_{2}, s_{3}> \\
<s_{5}, s_{1}, s_{2}><s_{5}, s_{1}, s_{2}><s_{5}, s_{4}, s_{2}> \\
<s_{6}, s_{1}, s_{1}><s_{5}, s_{1}, s_{1}><s_{5}, s_{2}, s_{3}>
\end{array}\right], \\
D^{3}=\left[\begin{array}{c}
D_{1}^{3} \\
D_{2}^{3} \\
D_{3}^{3} \\
D_{4}^{3}
\end{array}\right]=\left[\begin{array}{l}
<s_{7}, s_{3}, s_{4}><s_{7}, s_{3}, s_{3}><s_{5}, s_{2}, s_{5}> \\
<s_{6}, s_{3}, s_{4}><s_{5}, s_{1}, s_{2}><s_{6}, s_{2}, s_{3}> \\
<s_{7}, s_{2}, s_{4}><s_{6}, s_{1}, s_{2}><s_{7}, s_{2}, s_{4}> \\
<s_{7}, s_{2}, s_{3}><s_{5}, s_{2}, s_{1}><s_{6}, s_{1}, s_{1}>
\end{array}\right] .
\end{gathered}
$$

Then, the developed MAGDM approach can be applied to this decision-making problem using the following steps:

Step 1: We establish the LNN matrix $H^{*}=\left(h_{i, j}^{*}\right)_{4 \times 3}$ of ideal alternatives (ideal solutions) $H_{i}^{*}(i=1,2,3,4)$ as follows:

$$
H^{*}=\left[\begin{array}{c}
H_{1}^{*} \\
H_{2}^{*} \\
H_{3}^{*} \\
H_{4}^{*}
\end{array}\right]=\left[\begin{array}{c}
<s_{8}, s_{0}, s_{0}><s_{8}, s_{0}, s_{0}><s_{8}, s_{0}, s_{0}> \\
<s_{8}, s_{0}, s_{0}><s_{8}, s_{0}, s_{0}><s_{8}, s_{0}, s_{0}> \\
<s_{8}, s_{0}, s_{0}><s_{8}, s_{0}, s_{0}><s_{8}, s_{0}, s_{0}> \\
<s_{8}, s_{0}, s_{0}><s_{8}, s_{0}, s_{0}><s_{8}, s_{0}, s_{0}>
\end{array}\right],
$$

where $h_{i, j}^{*}=<\max \left(s_{\alpha_{i, j}}\right), \min \left(s_{\beta_{i, j}}\right), \min \left(s_{\gamma_{i, j}}\right)>=<s_{8}, s_{0}, s_{0}>(i=1,2,3,4 ; j=1,2,3)$ and $H_{k}^{*}=\left\{<s_{8}, s_{0}, s_{0}><s_{8}, s_{0}, s_{0}><s_{8}, s_{0}, s_{0}>\right\}(k=1,2,3,4)$.

Step 2: We calculate the weighted cosine measure values based on the distance between $D_{i}^{k}$ and the ideal alternative $H_{\mathrm{i}}^{*}$ by Equation (3) as follows: $0.9381,0.9554\} ;$

$C^{\omega 1}{ }_{L N N s}\left(D^{2}, H^{*}\right)=\left\{C^{\omega 1}{ }_{L N N S}\left(D_{1}^{2}, H_{1}^{*}\right), C^{\omega 1}{ }_{L N N S}\left(D_{2}^{2}, H_{2}^{*}\right), C^{\omega 1}{ }_{L N N s}\left(D_{3}^{2}, H_{3}^{*}\right), C^{\omega 1}{ }_{L N N s}\left(D_{4}^{2}, H_{4}^{*}\right)\right\}=\{0.9055,0.8974$, $0.8869,0.9212\} ;$ 
$C^{\omega 1}{ }_{L N N S}\left(D^{3}, H^{*}\right)=\left\{C^{\omega 1}{ }_{L N N S}\left(D_{1}^{3}, H_{1}^{*}\right), C^{\omega 1}{ }_{L N N S}\left(D_{2}^{3}, H_{2}^{*}\right), C^{\omega 1}{ }_{L N N S}\left(D_{3}^{3}, H_{3}^{*}\right), C^{\omega 1}{ }_{L N N s}\left(D_{4}^{3}, H_{4}^{*}\right)\right\}=\{0.8447,0.8807$, $0.9094,0.9407\}$

Similarly, we can calculate the weighted cosine measure values based on the included angle cosine of two vectors between $D_{\mathrm{i}}^{\mathrm{k}}$ and the ideal alternative $H_{\mathrm{i}}^{*}$ by Equation (4) as follows:

$C^{\omega 2}{ }_{L N N S}\left(D^{1}, H^{*}\right)=\left\{C^{\omega 2}{ }_{L N N s}\left(D_{1}^{1}, H_{1}^{*}\right), C^{\omega 2}{ }_{L N N S}\left(D_{2}^{1}, H_{2}^{*}\right), C^{\omega 2}{ }_{L N N s}\left(D_{3}^{1}, H_{3}^{*}\right), C^{\omega 2}{ }_{L N N s}\left(D_{4}^{1}, H_{4}^{*}\right)\right\}=\{0.9279,0.9464$, $0.9234,0.9367\}$;

$C^{\omega 2}{ }_{L N N s}\left(D^{2}, H^{*}\right)=\left\{C^{\omega 2}{ }_{L N N S}\left(D_{1}^{2}, H_{1}^{*}\right), C^{\omega 2}{ }_{L N N S}\left(D_{2}^{2}, H_{2}^{*}\right), C^{\omega 2}{ }_{L N N s}\left(D_{3}^{2}, H_{3}^{*}\right), C^{\omega 2}{ }_{L N N s}\left(D_{4}^{2}, H_{4}^{*}\right)\right\}=\{0.8684,0.8516$, $0.8459,0.9057\}$;

$C^{\omega 2}{ }_{L N N S}\left(D^{3}, H^{*}\right)=\left\{C^{\omega 2}{ }_{L N N S}\left(D_{1}^{3}, H_{1}^{*}\right), C^{\omega 2}{ }_{L N N S}\left(D_{2}^{3}, H_{2}^{*}\right), C^{\omega 2}{ }_{L N N s}\left(D_{3}^{3}, H_{3}^{*}\right), C^{\omega 2}{ }_{L N N s}\left(D_{4}^{3}, H_{4}^{*}\right)\right\}=\{0.7708,0.8400$, $0.8663,0.9287\}$.

Step 3: Considering the corresponding weight $\omega_{E}=(0.37,0.33,0.3)^{\mathrm{T}}$ of the experts to evaluate the alternatives $H_{i}(i=1,2,3,4)$, we can calculate the overall weighted cosine measure values based on distance by Equation (5) as follows:

$$
\begin{aligned}
& C^{\omega 1}{ }_{L N N s}\left(D^{k}, H_{1}\right)=0.37 \times C^{\omega 1}{ }_{L N N s}\left(D_{1}^{1}, H_{1}^{*}\right)+0.33 \times C^{\omega 1}{ }_{L N N s}\left(D_{1}^{2}, H_{1}^{*}\right)+0.3 \times C^{\omega 1}{ }_{L N N s}\left(D_{1}^{3}, H_{1}^{*}\right)=0.9009 ; \\
& C^{\omega 1}{ }_{L N N s}\left(D^{k}, H_{2}\right)=0.37 \times C^{\omega 1}{ }_{L N N s}\left(D_{1}^{1}, H_{2}^{*}\right)+0.33 \times C^{\omega 1}{ }_{L N N s}\left(D_{1}^{2}, H_{2}^{*}\right)+0.3 \times C^{\omega 1}{ }_{L N N s}\left(D_{1}^{3}, H_{2}^{*}\right)=0.9158 ; \\
& C^{\omega 1}{ }_{L N N s}\left(D^{k}, H_{3}\right)=0.37 \times C^{\omega 1}{ }_{L N N s}\left(D_{1}^{1}, H_{3}^{*}\right)+0.33 \times C^{\omega 1}{ }_{L N N s}\left(D_{1}^{2}, H_{3}^{*}\right)+0.3 \times C^{\omega 1}{ }_{L N N s}\left(D_{1}^{3}, H_{3}^{*}\right)=0.9126 ; \\
& C^{\omega 1}{ }_{L N N s}\left(D^{k}, H_{4}\right)=0.37 \times C^{\omega 1}{ }_{L N N s}\left(D_{1}^{1}, H_{4}^{*}\right)+0.33 \times C^{\omega 1}{ }_{L N N s}\left(D_{1}^{2}, H_{4}^{*}\right)+0.3 \times C^{\omega 1}{ }_{L N N s}\left(D_{1}^{3}, H_{4}^{*}\right)=0.9397 .
\end{aligned}
$$

Similarly, we can calculate the overall weighted cosine measure values based on the included angle cosine of two vectors by Equation (6) as follows:

$$
C^{\omega 2}{ }_{L N N s}\left(D^{k}, H_{1}\right)=0.8611, C^{\omega 2}{ }_{L N N s}\left(D^{k}, H_{2}\right)=0.8832, C^{\omega 2}{ }_{L N N s}\left(D^{k}, H_{3}\right)=0.8807, C^{\omega 2}{ }_{L N N s}\left(D^{k}, H_{4}\right)=0.9241 .
$$

Step 4: According to the above values of $C^{\omega 1}{ }_{L N N s}\left(D^{k}, H_{i}\right)$ and $C^{\omega 2}{ }_{L N N s}\left(D^{k}, H_{i}\right)(i=1,2,3,4)$, both the cosine measure values based on distance and the cosine measure values based on the included angle cosine of two vectors, there are the same ranking orders: $H_{4}>H_{2}>H_{3}>H_{1}$. Thus, according to the maximum value of cosine similarity measures, the alternative $H_{4}$ is the best choice.

\subsection{Related Comparison}

For further comparison, Table 1 lists the MAGDM results based on the cosine measures of LNNs proposed in this paper and the LNNWAA and LNNWGA Operators in the relevant paper [48], respectively.

Obviously, from the result of Table 1, ranking orders and the best alternatives based on the new method proposed in this paper are consistent with the results provided by Fang and Ye [48]. Compared with the literature [48], the calculation process of the cosine measures for MAGDM proposed in this paper is relatively simple compared to the LNNWGA operator and the LNNWAA operator in [48]. For further comparison, the MAGDM methods developed in the relevant papers [43,44] cannot deal with indeterminate and inconsistent linguistic information; while the method presented in this paper can solve linguistic decision-making problems with LNN information. The above comparisons demonstrate that this paper presented a new way for solving decision-making problems under an LNN environment.

Table 1. Decision results based on LNN MAGDM methods.

\begin{tabular}{cccc}
\hline MAGDM Method & Cosine Measure Value (Score Function) & Ranking Order & The Best Alternative \\
\hline$C^{\omega 1}{ }_{L N N s}\left(D^{k}, H_{i}\right)$ & $0.9009,0.9158,0.9126,0.9397$ & $H_{4}>H_{2}>H_{3}>H_{1}$ & $H_{4}$ \\
$C^{\omega 2}{ }_{L N N s}\left(D^{k}, H_{i}\right)$ & $0.8611,0.8832,0.8807,0.9241$ & $H_{4}>H_{2}>H_{3}>H_{1}$ & $H_{4}$ \\
LNNWAA Operator [48] & $0.7528,0.7770,0.7613,0.8060$ & $H_{4}>H_{2}>H_{3}>H_{1}$ & $H_{4}$ \\
LNNWGA Operator [48] & $0.7397,0.7747,0.7513,0.8035$ & $H_{4}>H_{2}>H_{3}>H_{1}$ & $H_{4}$ \\
\hline
\end{tabular}




\section{Conclusions}

Under a linguistic environment, two cosine similarity measures of LNNs based on the distance and the included angle cosine of two vectors were presented in this paper. Then, an MAGDM method with LNNs was developed based on the proposed cosine similarity measures. Finally, to demonstrate the application and effectiveness of the proposed method, we introduced a practical example about the MAGDM problems based on the cosine similarity measures of LNNs. The decision-making results show that the proposed method can effectively solve decision-making problems with LNN information. In the future work, we shall study some new correlation coefficients between LNNs and their MAGDM methods, and extend the similarity measures to linguistic neutrosophic cubic numbers [51].

Acknowledgments: This paper was supposed by the Public Technology Research Project of Zhejiang province (LGG18F030008).

Author Contributions: Lilian Shi proposed two cosine measures of LNNs and their decision-making method; Jun Ye revised the manuscript; the authors have read and approved the final manuscript.

Conflicts of Interest: The author declares that there is no conflict of interest regarding the publication of this paper.

\section{References}

1. Zadeh, L.A. The concept of a linguistic variable and its application to approximate reasoning Part I. Inf. Sci. 1975, 8, 199-249. [CrossRef]

2. Herrera, F.; Herrera-Viedma, E.; Verdegay, L. A model of consensus in group decision making under linguistic assessments. Fuzzy Sets Syst. 1996, 79, 73-87. [CrossRef]

3. Herrera, F.; Herrera-Viedma, E. Linguistic decision analysis: Steps for solving decision problems under linguistic information. Fuzzy Sets Syst. 2000, 115, 67-82. [CrossRef]

4. Wang, J.H.; Hao, J.Y. A new version of 2-tuple fuzzy linguistic representation model for computing with words. IEEE Trans. Fuzzy Syst. 2006, 14, 435-445. [CrossRef]

5. Martı'nez, L.; Herrera, F. An overview on the 2-tuple linguistic model for computing with words in decision making: Extensions, applications and challenges. Inf. Sci. 2012, 207, 1-18. [CrossRef]

6. José, M.M.; Anna, M.G.-L. Induced 2-tuple linguistic generalized aggregation operators and their application in decision-making. Inf. Sci. 2013, 236, 1-16. [CrossRef]

7. Wang, J.; Wang, J.Q.; Zhang, H.Y.; Chen, X.H. Multi-criteria group decision making approach based on 2-tuple linguistic aggregation operators with multi-hesitant fuzzy linguistic information. Int. J. Fuzzy Syst. 2016, 18, 81-97. [CrossRef]

8. Liu, P.D.; Teng, F. An extended TODIM method for multiple attribute group decision-making based on 2-dimension uncertain linguistic variable. Complexity 2016, 21, 20-30. [CrossRef]

9. Liu, P.D.; He, L.; Yu, X. Generalized hybrid aggregation operators based on the 2-dimension uncertain linguistic information for multiple attribute group decision making. Group Decis. Negot. 2016, 25, $103-126$. [CrossRef]

10. Liu, W.H.; Liu, H.B.; Li, L.L. A multiple attribute group decision making method based on 2-dimension uncertain linguistic weighted Heronian mean aggregation operator. Int. J. Comput. Commun. Control 2017, 12, 254-264. [CrossRef]

11. Xu, Z.S. A method based on linguistic aggregation operators for group decision making with linguistic preference relations. Inf. Sci. 2004, 166, 19-30. [CrossRef]

12. Xu, Z.S. A note on linguistic hybrid arithmetic averaging operator in multiple attribute group decision making with linguistic information. Group Decis. Negot. 2006, 15, 593-604. [CrossRef]

13. Martı́nez, L.; Da, R.; Herrera, F.; Herrera-Viedma, E.; Wang, P.P. Linguistic decision making: Tools and applications. Inf. Sci. 2009, 179, 2297-2298. [CrossRef]

14. Merigo', J.M.; Casanovas, M.; Martı'nez, L. Linguistic aggregation operators for linguistic decision making based on the Dempster-Shafer theory of evidence. Int. J. Uncertain. Fuzz. Knowl.-Based Syst. 2010, 18, $287-304$. [CrossRef]

15. Pei, Z.; Shi, P. Fuzzy risk analysis based on linguistic aggregation operators. Int. J. Innov. Comput. Inf. Control 2011, 7, 7105-7117. 
16. Wang, J.Q.; Li, H.B. Multi-criteria decision-making method based on aggregation operators for intuitionistic linguistic fuzzy numbers. Control Decis. 2010, 25, 1571-1574.

17. Du, Y.; Zuo, J. An extended TOPSIS method for the multiple attribute group decision making problems based on intuitionistic linguistic numbers. Sci. Res. Essays 2011, 19, 4125-4132.

18. Chen, Z.C.; Liu, P.D.; Pei, Z. An approach to multiple attribute group decision making based on linguistic intuitionistic fuzzy numbers. Int. J. Comput. Intell. Syst. 2015, 8, 747-760. [CrossRef]

19. Liu, P.D. Some generalized dependent aggregation operators with intuitionistic linguistic numbers and their application to group decision making. J. Comput. Syst. Sci. 2013, 79, 131-143. [CrossRef]

20. Ju, Y.B.; Liu, X.Y.; Ju, D.W. Some new intuitionistic linguistic aggregation operators based on Maclaurin symmetric mean and their applications to multiple attribute group decision making. Soft Comput. 2015, 20, 4521-4548. [CrossRef]

21. Yu, S.M.; Wang, J.; Wang, J.Q. An extended TODIM approach with intuitionistic linguistic numbers. Int. Trans. Oper. Res. 2016. [CrossRef]

22. Zhang, H.Y.; Peng, H.G.; Wang, J.; Wang, J.Q. An extended outranking approach for multi-criteria decision-making problems with linguistic intuitionistic fuzzy numbers. Appl. Soft Comput. 2017, 59, 462-474. [CrossRef]

23. Liu, P.; Wang, P. Some improved linguistic intuitionistic fuzzy aggregation operators and their applications to multiple-attribute decision making. Int. J. Inf. Technol. Decis. Mak. 2017, 16, 817-850. [CrossRef]

24. Wang, J.Q.; Wang, P.; Wang, J.; Zhang, H.Y.; Chen, X.H. Atanassov's interval-valued intuitionistic linguistic multi-criteria group decision-making method based on trapezium cloud model. IEEE Trans. Fuzzy Syst. 2015, 23, 542-554. [CrossRef]

25. Rodriguez, R.M.; Martinez, L.; Herrera, F. Hesitant fuzzy linguistic term sets for decision making. IEEE Trans. Fuzzy Syst. 2012, 20, 109-119. [CrossRef]

26. Liu, H.B.; Rodrı'guez, R.M. A fuzzy envelope for hesitant fuzzy linguistic term set and its application to multi-criteria decision making. Inf. Sci. 2014, 258, 220-238. [CrossRef]

27. Wang, J.Q.; Wu, J.T.; Wang, J.; Zhang, H.Y.; Chen, X.H. Interval valued hesitant fuzzy linguistic sets and their applications in multi-criteria decision-making problems. Inf. Sci. 2014, 288, 55-72. [CrossRef]

28. Meng, F.Y.; Chen, X.H.; Zhang, Q. Multi-attribute decision analysis under a linguistic hesitant fuzzy environment. Inf. Sci. 2014, 267, 287-305. [CrossRef]

29. Wang, J.; Wang, J.Q.; Zhang, H.Y.; Chen, X.H. Multi-criteria decision-making based on hesitant fuzzy linguistic term sets: An outranking approach. Knowl.-Based Syst. 2015, 86, 224-236. [CrossRef]

30. Wang, J.Q.; Wu, J.T.; Wang, J.; Zhang, H.Y.; Chen, X.H. Multi-criteria decision-making methods based on the Hausdorff distance of hesitant fuzzy linguistic numbers. Soft Comput. 2015, 20, 1621-1633. [CrossRef]

31. Wang, J.; Wang, J.Q.; Zhang, H.Y. A likelihood-based TODIM approach based on multi-hesitant fuzzy linguistic information for evaluation in logistics outsourcing. Comput. Ind. Eng. 2016, 99, 287-299. [CrossRef]

32. Zhou, H.; Wang, J.Q.; Zhang, H.Y.; Chen, X.H. Linguistic hesitant fuzzy multi-criteria decision-making method based on evidential reasoning. Int. J. Syst. Sci. 2016, 47, 314-327. [CrossRef]

33. Tüysüz, F.; Şimşek, B. A hesitant fuzzy linguistic term sets-based AHP approach for analyzing the performance evaluation factors: An application to cargo sector. Complex Intell. Syst. 2017. [CrossRef]

34. Gou, X.; Liao, H.C.; Xu, Z.S.; Herrera, F. Double hierarchy hesitant fuzzy linguistic term set and MULTIMOORA method: A case of study to evaluate the implementation status of haze controlling measures. Inf. Fusion 2017, 38, 22-34. [CrossRef]

35. Zhou, H.; Wang, J.; Li, X.E.; Wang, J.Q. Intuitionistic hesitant linguistic sets and their application in multi-criteria decision-making problems. Oper. Res. Int. J. 2016, 16, 131-160. [CrossRef]

36. Faizi, S.; Rashid, T.; Zafar, S. An outranking method for multi-criteria group decision making using hesitant intuitionistic fuzzy linguistic term sets. J. Intell. Fuzzy Syst. 2017, 32, 2153-2164. [CrossRef]

37. Smarandache, F. Neutrosophy: Neutrosophic Probability, Set, and Logic; American Research Press: Rehoboth, DE, USA, 1998.

38. Smarandache, F. Introduction to Neutrosophic Measure, Neutrosophic Integral, and Neutrosophic Probability; Sitech and Education Publishing: Craiova, Romania; Columbus, OH, USA, 2013.

39. Smarandache, F. Introduction to Neutrosophic Statistics; Sitech and Education Publishing: Craiova, Romania; Columbus, OH, USA, 2014. 
40. Liang, R.X.; Wang, J.Q.; Zhang, H.Y. Evaluation of e-commerce websites: An integrated approach under a single-valued trapezoidal neutrosophic environment. Knowl.-Based Syst. 2017, in press. [CrossRef]

41. Nie, R.X.; Wang, J.Q.; Zhang, H.Y. Solving solar-wind power station location problem using an extended WASPAS technique with Interval neutrosophic sets. Symmetry 2017, 9, 106. [CrossRef]

42. Wang, L.; Zhang, H.Y.; Wang, J.Q. Frank choquet bonferroni mean operators of bipolar neutrosophic sets and their application to multi-criteria decision-making problems. Int. J. Fuzzy Syst. 2017. [CrossRef]

43. Ye, J. Bidirectional projection method for multiple attribute group decision making with neutrosophic numbers. Neural Comput. Appl. 2017, 28, 1021-1029. [CrossRef]

44. Ye, J. Multiple-attribute group decision-making method under a neutrosophic number environment. J. Intell. Syst. 2016, 25, 377-386. [CrossRef]

45. Li, Y.Y.; Zhang, H.; Wang, J.Q. Linguistic neutrosophic sets and their application in multicriteria decision-making problems. Int. J. Uncertain. Quantif. 2017, 7, 135-154. [CrossRef]

46. Luo, S.Z.; Cheng, P.F.; Wang, J.Q.; Huang, Y.J. Selecting project delivery systems based on simplified neutrosophic linguistic preference relations. Symmetry 2017, 9, 151. [CrossRef]

47. Wang, J.Q.; Yang, Y.; Li, L. Multi-criteria decision-making method based on single valued neutrosophic linguistic Maclaurin symmetric mean operators. Neural Comput. Appl. 2016. [CrossRef]

48. Fang, Z.B.; Ye, J. Multiple attribute group decision-making method based on linguistic neutrosophic numbers. Symmetry 2017, 9, 111. [CrossRef]

49. Biswas, P.; Pramanik, S.; Giri, B.C. Cosine Similarity Measure Based Multi-attribute Decision-making with Trapezoidal Fuzzy Neutrosophic Numbers. Neutrosophic Sets Syst. 2014, 8, 47-57. [CrossRef]

50. Mahmood, T.; Ye, J.; Khan, Q. Vector similarity measures for simplified neutrosophic hesitant fuzzy set and their applications. J. Inequal. Spec. Funct. 2016, 7, 176-194.

51. Ye, J. Linguistic neutrosophic cubic numbers and their multiple attribute decision-making method. Information 2017, 8, 110. [CrossRef]

(C) 2017 by the authors. Licensee MDPI, Basel, Switzerland. This article is an open access article distributed under the terms and conditions of the Creative Commons Attribution (CC BY) license (http:/ / creativecommons.org/licenses/by/4.0/). 